\title{
Use of Pharmacist Consultations for Nonprescription Laxatives in Japan: An Online Survey
}

\author{
Keita Shibata, ${ }^{* a}$ Arisa Matsumoto, ${ }^{a}$ Ayumi Nakagawa, ${ }^{a}$ Keiko Akagawa, ${ }^{a}$ Akihiro Nakamura,${ }^{b}$ \\ Toshinori Yamamoto, ${ }^{c}$ and Naomi Kurata ${ }^{a}$ \\ ${ }^{a}$ Division of Community Healthcare and Pharmacy, Department of Healthcare and Regulatory Sciences, School of \\ Pharmacy, Showa University; 1-5-8 Hatanodai, Shinagawa-ku, Tokyo 142-8555, Japan: ${ }^{b}$ Division of Pharmaceutical \\ Sciences, Department of Pharmacotherapeutics, School of Pharmacy, Showa University; 1-5-8 Hatanodai, \\ Shinagawa-ku, Tokyo 142-8555, Japan: and ${ }^{c}$ Division of Clinical Pharmacy, Department of Pharmacotherapeutics, \\ School of Pharmacy, Showa University; 1-5-8 Hatanodai, Shinagawa-ku, Tokyo 142-8555, Japan. \\ Received January 5, 2016; accepted August 22, 2016
}

Community pharmacies in Japan have long been advocated as effective sources of nonprescription medicines and health-related advice. Consumers sometimes self-treat symptoms of minor illnesses without consulting a pharmacist because the benefits of such consultations are not adequately recognized. The aim of this study was to investigate the use and impact of pharmacist consultations before purchase of nonprescription laxatives. An online survey was conducted July 14-22, 2012 with 500 respondents (250 men, 250 women), ranging 20-60 years old. All participants had purchased nonprescription laxatives for constipation within the past year. Stratified analysis was used to compare responses in groups that had and had not consulted a pharmacist before purchase. Consulting a pharmacist appears to improve consumers' awareness and makes them more likely to use appropriate medication. Those who consulted a pharmacist were better able to identify side effects and take appropriate action than the group that did not consult the pharmacist. Those who consulted a pharmacist were also significantly more likely to say that they would consult a pharmacist in the future. These results indicate that it is important for consumers to be able to consult with pharmacists, to improve consumers' awareness of side effects and to self-medicate appropriately, and hence improve their quality of life. Pharmacists in community pharmacy could be more active in health promotion campaigns, such as drug safety, campaigns, to raise their public profile. Increased public awareness of what pharmacists in community pharmacy do will make it easier for patients to consult with them.

Key words pharmacist consultation; nonprescription laxative; constipation; self-medication

In Japan, most health services are provided through the National Health Insurance system, which covers the country's entire population. There is a fixed cost-sharing rate that varies with age and a general upper limit on monthly out-of-pocket spending for all age groups.

Japan's population is aging at an unprecedentedly rapid pace. The proportion of the population aged 65 or over in 2014 was $26.0 \%$, the highest in the world. ${ }^{1)}$ This has resulted in an increase in national medical expenses. Policy-makers in Japan are considering how to provide medical care in the future without huge spending increases.

The Japanese government approved the first version of the Japan Revitalization Strategy on June 14, 2013. ${ }^{2)}$ This document sets out plans to build a society with improved health and life expectancy by offering preventive healthcare services and lifestyle support. To achieve this goal, it is also important for pharmacies to provide health-related information, including advice on the proper use of nonprescription medicines. This should contribute to reduced medical expenses.

Since 2009, nonprescription medicines in Japan have been classified into three categories (Schedule I, II and III) depending on the degree of risk they present. Schedule I medicines can only be sold by pharmacists and require the provision of written information. A 2013 investigation by the Ministry of Health, Labour and Welfare of Japan found that only $67.5 \%$ of consumers who purchased nonprescription medicines were provided with this written information. ${ }^{3)}$ This suggests that the system has not completely taken root.
In the United Kingdom, it was investigated how important the pharmacist in health management is and reported about the customer's use of pharmacy in that study. ${ }^{4}$ Especially, it was also mentioned who had an influence on the customer's purchase for all OTC medicines. Almost half of those customers buying an OTC medicine for the first time cited the recommendation from pharmacist as being their influence on purchase. ${ }^{4)}$ In Northern Ireland, general attitudes towards the use of OTC medicines. ${ }^{5)}$ The factor which most frequently influenced the choice OTC medicines, was a recommendation by the pharmacist more than experience they used it before. ${ }^{5)}$ These finding indicated that pharmacist could be more proactive in the management of inappropriate OTC medicines use. On the other hand, in Japanese investigation, it was reported that $30-40 \%$ of customers cited the recommendation from pharmacist to purchase OTC medicine. ${ }^{6-8)}$ In addition, Narui et al. reported that the proportion of the Japanese population choosing to self-medicate was only $46.3 \% .^{9)}$ They suggested that more self-medication was possible. A report by the Ministry of Health, Labour and Welfare of Japan found that $32.3 \%$ of people acquired information about nonprescription medicines from a face-to-face consultation with a pharmacist in community pharmacy, and $9.5 \%$ chose to purchase medicines in a pharmacy because they could consult the pharmacist if they wished to do so. ${ }^{10)}$ However, more than half of consumers felt that it was not useful to consult pharmacists or obtain information from them.

It is therefore important for consumers to know that face- 
to-face consultations with pharmacists in community pharmacy are available. In this study, we focused on laxatives because they are not Schedule I medicines, and because selfmedication is the norm for the treatment of constipation. ${ }^{11,12}$ Our aim was to investigate the use and impact of face-to-face consultations with pharmacists in community pharmacy.

\section{MATERIALS AND METHODS}

Ethical Consideration This study was approved by the Ethics Committee of the Showa University School of Pharmacy (No. 173) and conducted in accordance with Japanese ethical guidelines for epidemiological research and the Declaration of Helsinki. All respondents gave informed consent.

Study Design An online survey was conducted in Japan in the period July 14-22, 2012. The respondents employed voluntary sampling technique to collect data. The online survey was employed randomly among the online research panel of 1700000 people, owned by Cross Marketing Inc. (Tokyo, Japan). Once the participants received the invitation to the online survey, they voluntarily took in part of the survey. A total of 500 participants (250 men, 250 women), ranging in age from 20 to 60 years old, and distributed equally across the age ranges, were recruited by Cross Marketing Inc. To evaluate the effect to simple constipation, older people who may have another disease were excluded. Younger people who are not adult in law in Japan (under 20) were also excluded because it is hard to get correct their consent as a research. Participants had all purchased nonprescription laxatives for constipation within the past year, and lived in Tokyo, Kanagawa, Chiba or Saitama prefecture. We excluded healthcare professionals or anyone with a family member working as a healthcare professional, and anyone who answered the questionnaire within $3 \mathrm{~min}$, because the average response time was $6 \mathrm{~min}$. The flow chart of participant recruitment is shown in Fig. 1. The employed total respondents were 8840 . 7645 were excluded because they did not match selection criteria and 1195 were assessed as eligible respondents. After that, the respondents narrowed down to 690 randomly. It was conducted by considering those who drop out in the middle or answer inappropriately.
Actually, 18 dropped out and 100 answered inappropriately and 572 answered till the end appropriately. Finally, 500 respondents assigned randomly as we designed. All identifying information was kept confidential and informed consent was obtained from each participant via the website. Table 1 lists the survey items.

Statistical Analysis All statistical analysis was performed with IBM SPSS v.22 (Tokyo, Japan). Stratified analysis was used to compare those who consulted a pharmacist in community pharmacy and those who did not. Statistical significance was evaluated using Fisher's exact test, with $p<0.05$ considered statistically significant.

\section{RESULTS}

Characteristics of Respondents In total, 78 (16\%) respondents had purchased laxatives after consulting with a

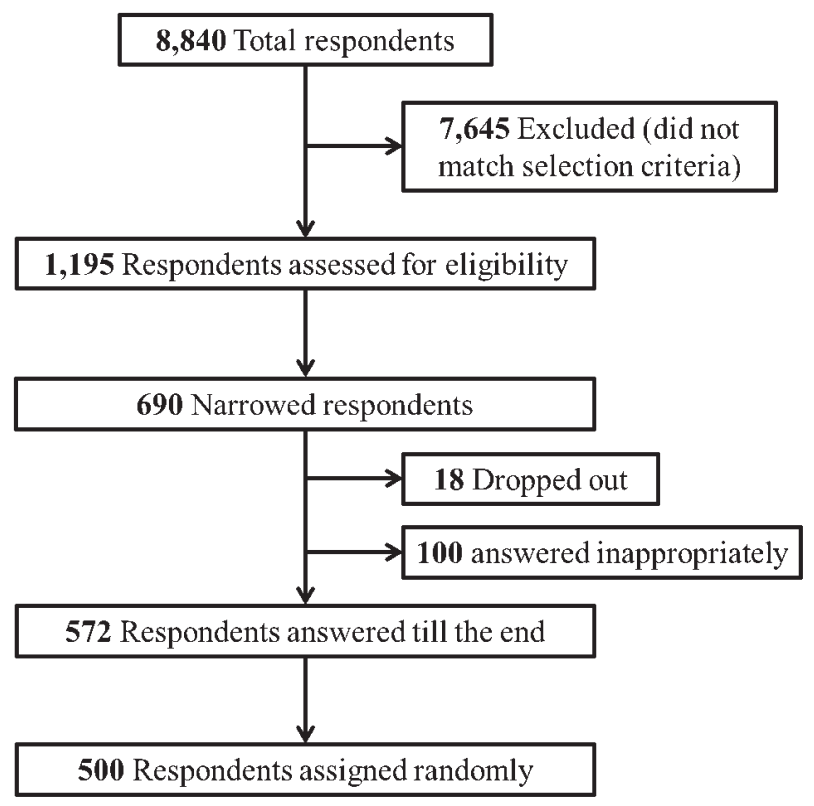

Fig. 1. The Flow Chart of Participant Recruitment

Table 1. List of Questions Used in the Survey

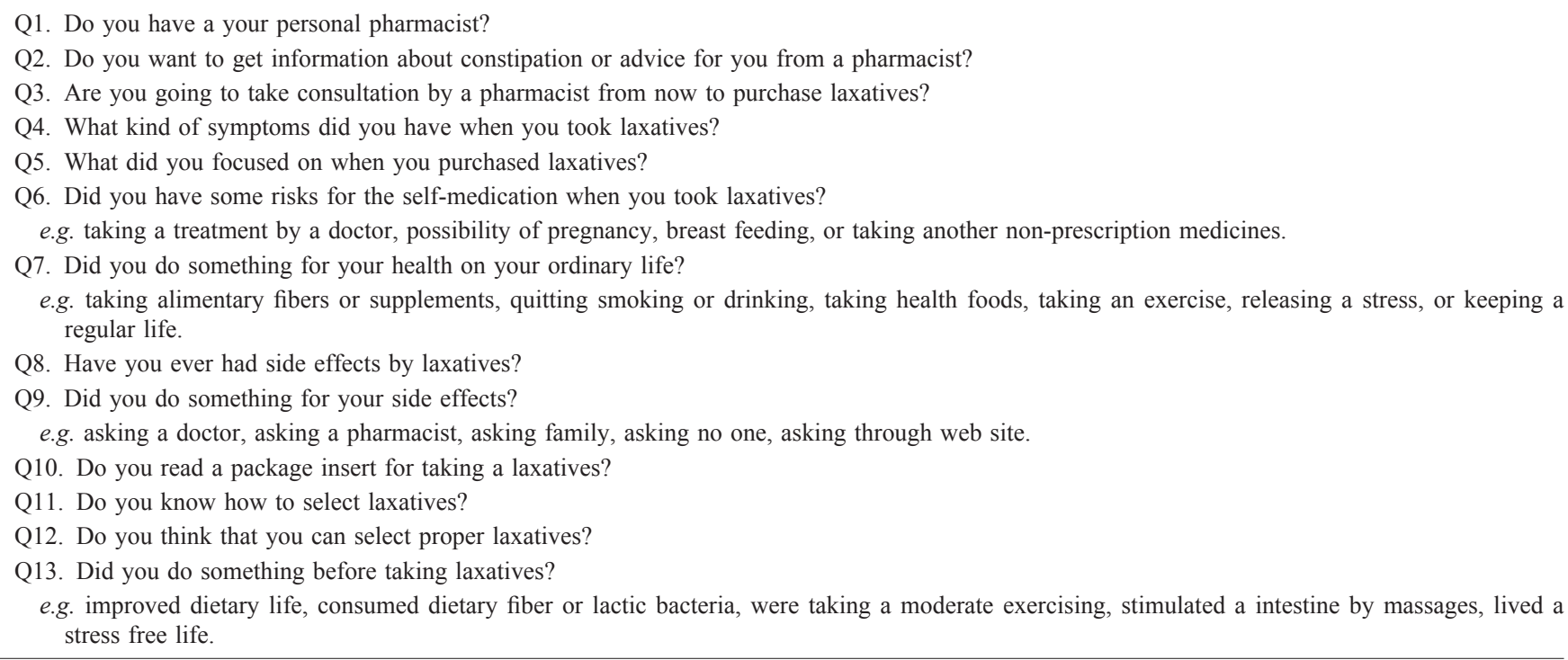


pharmacist in community pharmacy, and 422 (84\%) had purchased without consulting. Table 2 shows the distribution of sex, age and residence of the respondents who had purchased laxatives with and without a pharmacist consultation.

Relationship between the Patient and the Pharmacist in Community Pharmacy (Q1-3) The results regarding the relationship between patients and pharmacists in community pharmacy are shown in Fig. 2. The groups who purchased laxatives with and without a consultation with a pharmacist

Table 2. Characteristics of Respondents

\begin{tabular}{lccccc}
\hline \hline & Total & \multicolumn{2}{c}{ With consultation } & Without consultation \\
\hline Sex & $n$ & $n$ & $\%$ & $n$ & $\%$ \\
\hline Male & 250 & 42 & 17 & 208 & 83 \\
Female & 250 & 36 & 14 & 214 & 86 \\
\hline \multicolumn{1}{c}{ Total } & 500 & 78 & 16 & 422 & 84 \\
\hline Age & $n$ & $n$ & $\%$ & $n$ & $\%$ \\
\hline 20-29 & 100 & 12 & 12 & 88 & 88 \\
$30-39$ & 100 & 11 & 11 & 89 & 89 \\
$40-49$ & 100 & 13 & 13 & 87 & 87 \\
50-59 & 100 & 17 & 17 & 83 & 83 \\
$60-69$ & 100 & 25 & 25 & 75 & 75 \\
\hline Total & 500 & 78 & 16 & 422 & 84 \\
\hline Residence & $n$ & $n$ & $\%$ & $n$ & $\%$ \\
\hline Tokyo & 206 & 34 & 17 & 172 & 83 \\
Kanagawa & 136 & 15 & 11 & 121 & 89 \\
Chiba & 74 & 14 & 19 & 60 & 81 \\
Saitama & 84 & 15 & 18 & 69 & 82 \\
\hline Total & 500 & 78 & 16 & 422 & 84 \\
\hline
\end{tabular}
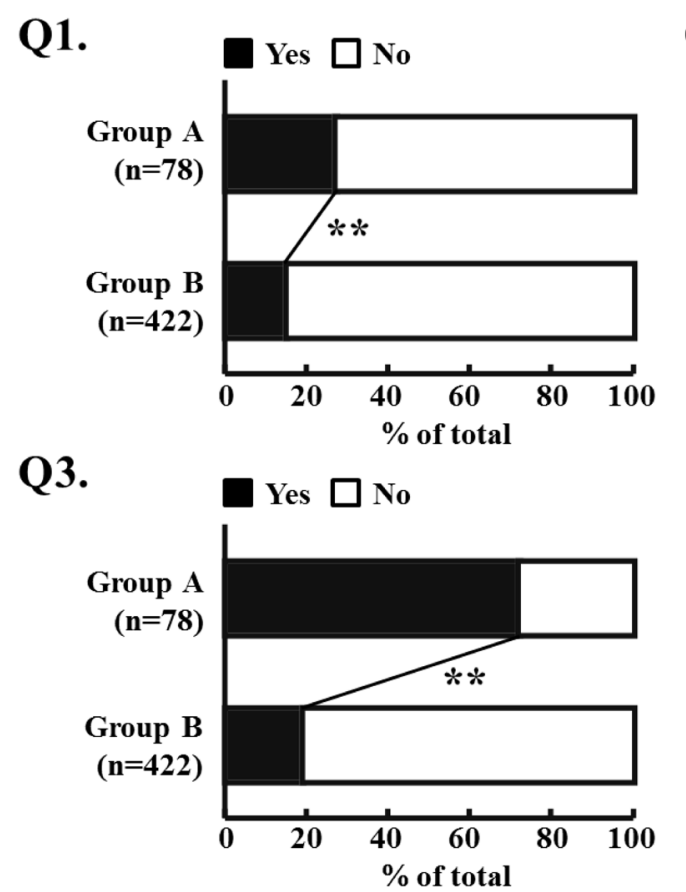

are shown as Groups A and B, respectively. It means people in Group B purchased it by themselves without pharmacist consultations.

In total, 83 respondents (17\%) said that they had a personal pharmacist (Fig. 2, Q1). The number of these in Group A was 21 (27\% of Group A) and in Group B was 62 (15\%). The percentage of respondents who had their personal pharmacist in Group A was significantly greater than that of Group B $(p<0.01)$. The number of respondents who wanted information or advice about constipation from a pharmacist was 361 (72\%), including 70 in Group A (90\%) and 291 in Group B $(69 \%)$ (Fig. 2, Q2). Again, the percentage was significantly higher in Group A $(p<0.01)$ than in Group B.

A total of 134 respondents (27\%) planned to consult a pharmacist before purchasing laxatives again (Fig. 2, Q3). Of these, there were 56 in Group A (72\%) and 78 in Group B (18\%). Again, the percentage in Group A was significantly higher $(p<0.01)$ than in Group B.

Patients' Situation and Symptoms before Taking Laxatives (Q4-9) Figures 3-5 show the results to questions about why patients took laxatives. Patients were asked about their symptoms before taking laxatives, including whether they had feelings of bloating, stomachache, nausea, difficulty defecating, feelings of incomplete evacuation, no urge to defecate, infrequent stool output, other symptoms, or no subjective symptoms. Respondents were allowed multiple answers (Fig. 3, Q4). The rate of those experiencing bloating, nausea, and difficulty defecating in Group A was significantly higher than in Group B $(p<0.05)$. In total, $314(63 \%)$ respondents cited bloating, $34(7 \%)$ experienced nausea, and 187 (37\%) had difficulty defecating. The numbers of those with bloating, nausea, and difficulty defecating in Group A were 59 (76\%), 10 $(13 \%)$ and $38(49 \%)$, respectively, and $255(60 \%), 24(6 \%)$ and $149(35 \%)$ in Group B.

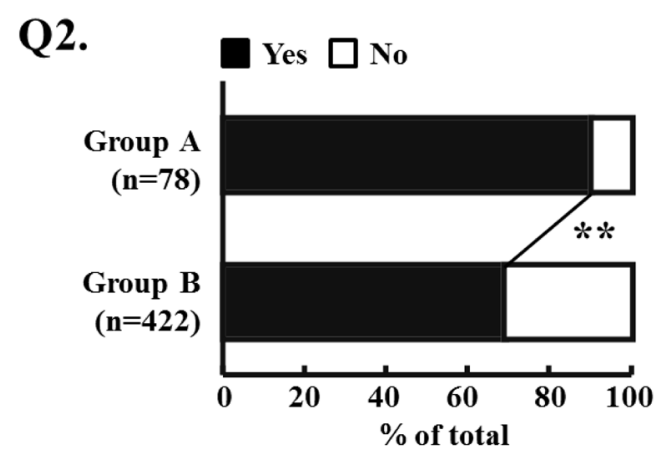

Fig. 2. Relationship between Patients and Pharmacists in Community Pharmacy (Q1-3)

Q1 asked whether patients had their own personal pharmacist. Q2 asked whether they wanted information or advice about constipation from a pharmacist. Q3 asked whether they planned to consult a pharmacist before purchasing laxatives in the future. Percentage of total numbers in each group is shown. $* * p<0.01$, statistically significant difference between Groups A and B. 
Q4. Group A (n=78) $\square$ Group B (n=422)

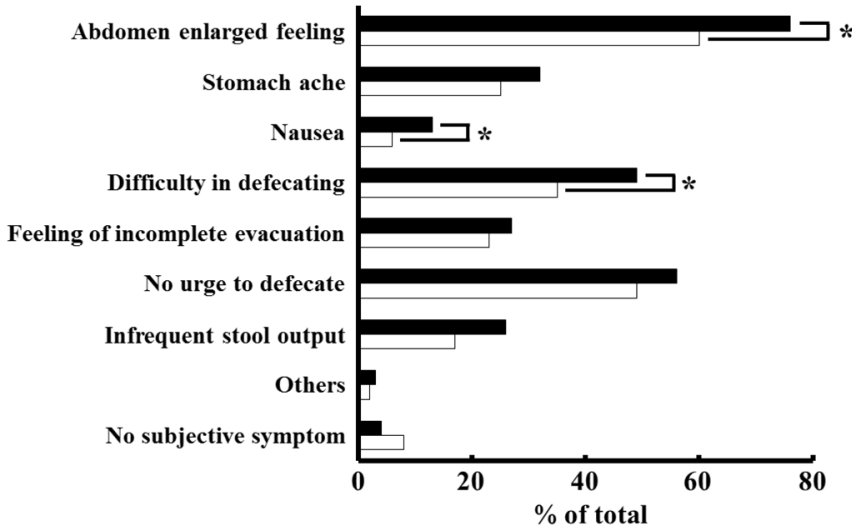

Fig. 3. Symptoms before Taking Laxatives (Q4)

Data show the rate in each group. ${ }^{*} p<0.05$, shows a statistically significant difference between Groups A and B.

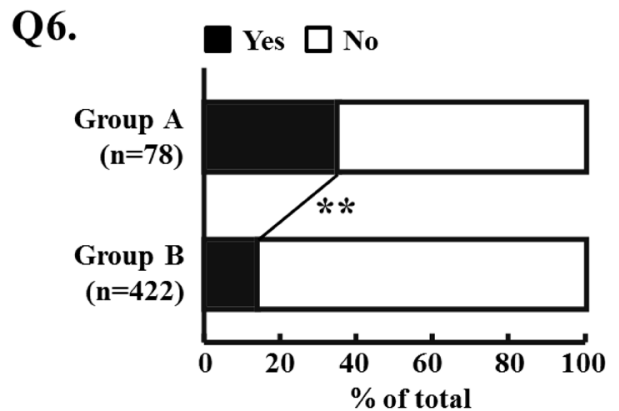

Q8.

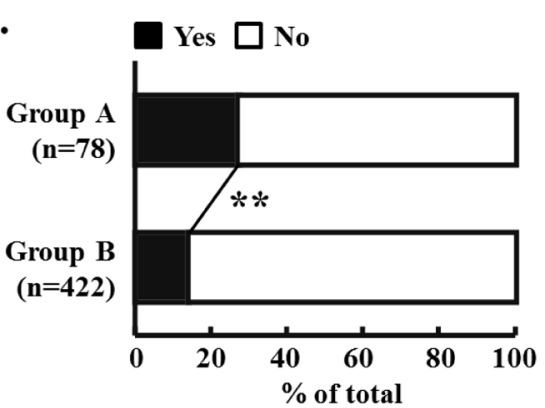

Q5.

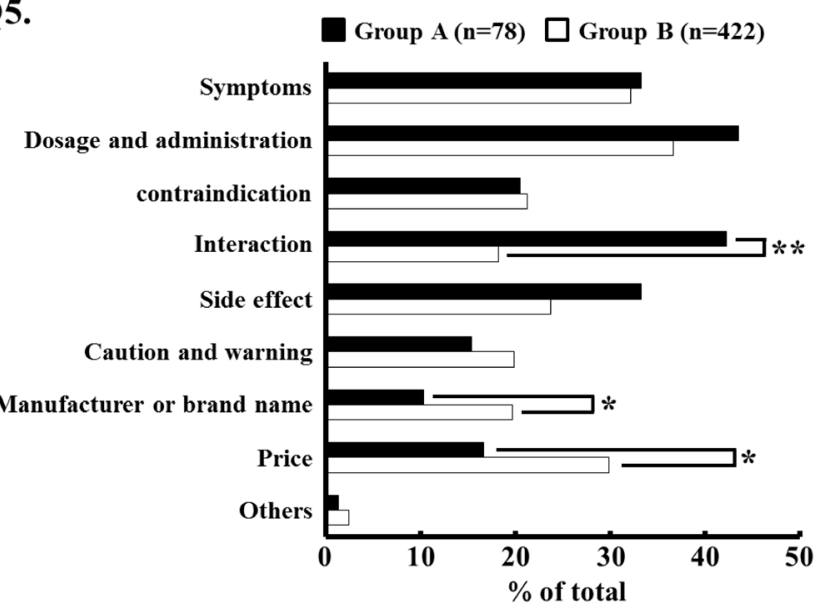

Fig. 4. What Patients Focused on When They Took Laxatives (Q5)

Data show the percentage chosen by respondents in each group. $* p<0.05$ and ** $p<0.01$, statistically significant differences between Groups A and B.

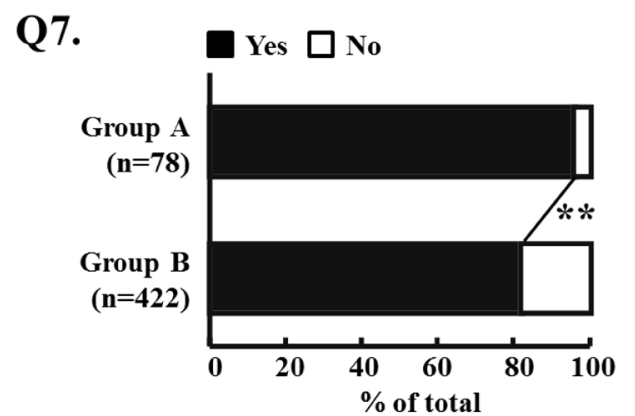

Q9.

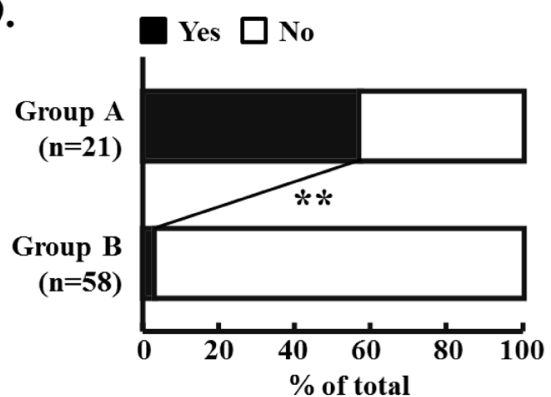

Fig. 5. Situations among Patients Taking Laxatives (Q6-9)

Q6 asked whether patients were at risk of complications when they took laxatives. Q7 asked whether they took steps to improve their health. Q8 asked whether they had ever had side effects from laxatives. Q9 asked whether they took appropriate action for their side effects. Data show the percentage of total numbers in each group. $*_{*} p<0.01$, statistically significant difference between Groups A and B.

Patients were asked what they focused on when selecting and taking laxatives among the following choices: symptoms, dosage and administration method, contraindications, interactions, side effects, cautions and warnings, manufacturer or brand name, price, or other factors. They were allowed multiple answers (Fig. 4, Q5). The percentage of those who answered "interaction" was significantly higher in group A, $33(42 \%)$ compared with $77(18 \%)$ in Group B $(p<0.01)$. The percentages of respondents who answered "manufacturer or brand name" and "price," however, were both significantly lower in group A $(p<0.05)$ than Group B. Eight $(10 \%)$ respondents vs. 83 (20\%) answered "brand name" and $13(17 \%) v s$. $126(30 \%)$ answered "price" in Groups A and B, respectively. In total, $110(22 \%)$ respondents reported focused on "interaction," 91 (18\%) "manufacturer or brand name," and 139 (28\%) on "price."
Self-medication using nonprescription medicines may have undesired side effects. Patients taking other medication, whether prescribed by a doctor or not, and women who may be pregnant or breast-feeding, are considered to be at risk when taking laxatives. A total of 87 respondents (17\%) were considered to be at risk because of the above factors (Fig. 5, Q6). Of these, $27(35 \%)$ and $60(14 \%)$ were in Groups A and $\mathrm{B}$, respectively. There were significantly more patients at risk in Group A $(p<0.01)$ than in Group B.

We asked patients whether they were taking dietary fiber or other supplements, quitting smoking or drinking, eating healthy foods, exercising, or taking steps to reduce stress, as these steps were regarded as important to improve health. Maintaining good health is also effective for preventing constipation. The total number in this category was $420(84 \%)$ (Fig. 5, Q7), with 75 (96\%) in Group A and 345 (82\%) in 


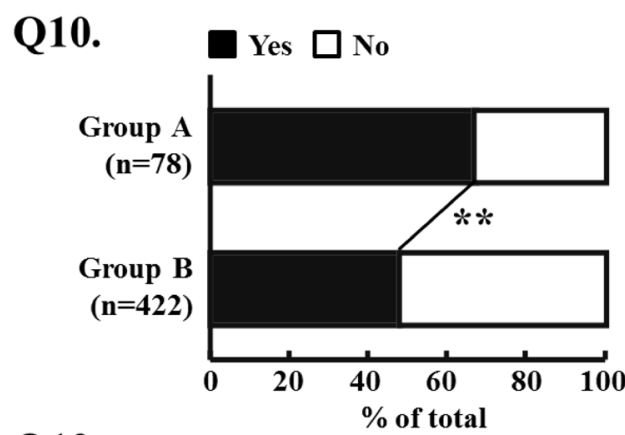

Q12.

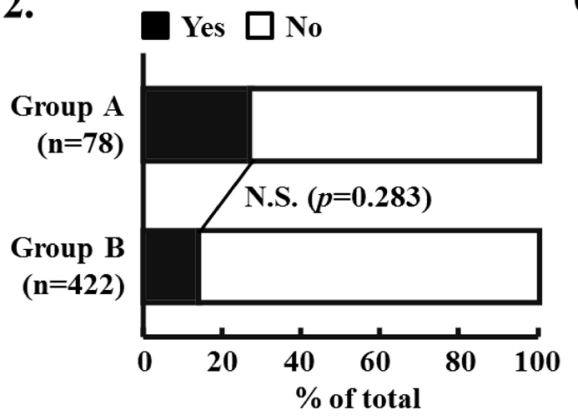

Q11.

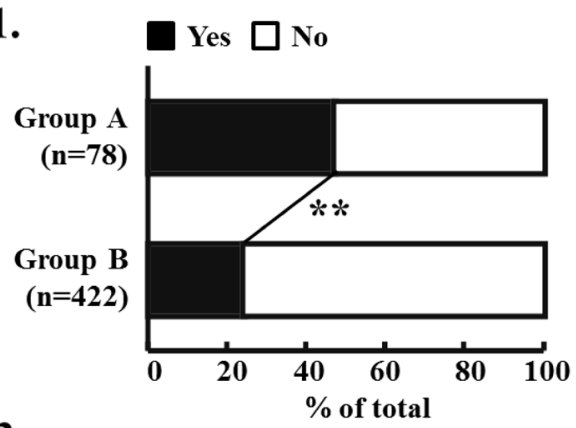

Q13.

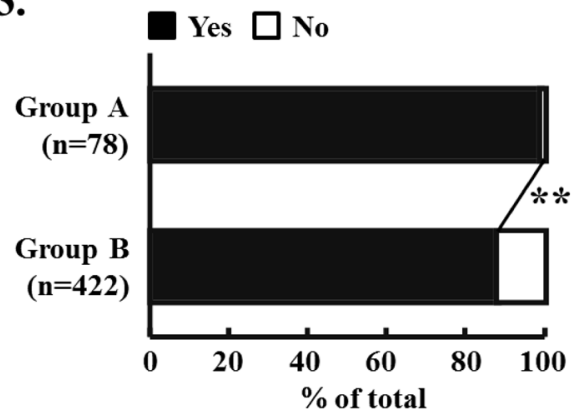

Fig. 6. Proper Usage of Laxatives (Q10-13)

Q10 asked whether patients read the package insert before taking laxatives. Q11 asked whether they knew how to select proper laxatives. Q12 asked whether they had in practice been able to select an appropriate laxative in fact. Q13 asked whether they took any action to improve their health before taking laxatives. Data show the percentages of total numbers in each group. ${ }^{* *} p<0.01$, statistically significant difference between Groups A and B.

Group B. Again, the percentage was significantly higher in Group A $(p<0.01)$ than Group B.

Patients should be aware of not only the desired effects of medication but also any possible side effects. These should be identified quickly to avoid negative effects on health. The total number of respondents who had ever experienced side effects from laxatives was 79 (16\%) (Fig. 5, Q8), of whom 21 $(27 \%)$ were in Group A and $58(14 \%)$ in Group B. Again, the percentage was significantly higher in Group A $(p<0.01)$ than Group B.

The action that patients take upon experiencing side effects from medication is important. Appropriate action in response to side effects from laxatives was considered to be asking a doctor or pharmacist for advice. Asking family or no one at all, or seeking advice via a website or social networking service was regarded as inappropriate action. In total, 14 respondents (18\%) took appropriate action (Fig. 5, Q9), of whom 12 (57\%) were in Group A and two (3\%) in Group B; again significantly more respondents appropriate in Group A $(p<0.01)$ took appropriate action than in Group B.

Proper Use of Laxatives (Q10-13) Figure 6 shows the results to questions about the proper usage of laxatives. It is important that patients check not only the box but also any inserts to understand how to correctly take medicines. The total number of responders who read an insert before taking laxatives was 256 (51\%) (Fig. 6, Q10), of whom 52 (67\%) were in Group A and 204 (48\%) in Group B. Again, this percentage was significantly higher for Group A $(p<0.01)$ than Group B.

Most respondents did not express confidence that they knew how to select an appropriate laxative. They therefore need to ask a professional, such as a pharmacist, to help them select an appropriate medicine. The total number of respondents who stated that they knew how to select laxatives was $138(28 \%)$ (Fig. 6, Q11), with $37(47 \%)$ in Group A and significantly less in Group B [101 (24\%)]. In contrast, only 79 (16\%) stated that they had actually been able to select an appropriate laxative (Fig. 6, Q12), with 21 (27\%) in Group A and $58(14 \%)$ in Group B, no significant difference $(p=0.283)$.

It may also be important to make lifestyle changes before taking medicines. We recorded the following lifestyle changes: improving diet, consuming dietary fiber or lactic bacteria, exercising moderately, stimulating the intestines using massage, or trying to reduce stress levels. In total, 450 respondents (90\%) changed their lifestyle (Fig. 6, Q13), of whom 77 (99\%) were in Group A and $373(88 \%)$ in Group B. Again, the percentage of respondents who made lifestyle changes was significantly higher in Group A $(p<0.01)$ than in Group B.

\section{DISCUSSION}

Because constipation is not life-threatening, the usual treatment is self-medication with nonprescription laxatives. ${ }^{11-13)}$ There is a wide range of sources of information about nonprescription medicines, including pharmacists, friends and family, books, magazines, the Internet, television, and advertisements. ${ }^{14)}$ It is important that people use laxatives appropriately, and pharmacists should be able to provide helpful professional advice to those seeking to treat constipation. Our results (Fig. 2) show that those who had consulted pharmacists in community pharmacy about laxatives (Group A) were more likely to have a personal pharmacist, to want information or advice about constipation from a pharmacist, and consult a pharmacist in the future. Therefore, for patients to more readily seek out effective consultation, it is helpful for them to establish good relationships with their local pharmacists. In addition, we should focus on what $69 \%$ of respondents in Group B wanted information or advice about constipation from a pharmacist but they purchased laxatives without a consultation with a pharmacist (Fig. 2, Q2). In the previous study, the most reasons not to have a consultation with a pharmacist 
were "Because I always take same medicine" and "Because I believe OTC medicines are safe." ${ }^{\text {(8) }}$ Because these are the result that they judged by themselves not to be any problem on it, there might be some people who did like that in the Group $\mathrm{B}$ in this study and they did not have a consultation with a pharmacist. Moreover, these judgements lead to the answer in Q3 and 89\% of respondents in the Group B are not going to take a consultation by a pharmacist from now to purchase laxatives (Fig. 2, Q3). From these, it is necessary for a pharmacist to support their self-medication to prevent a problem caused by such a judgement. For the effective treatment of constipation, it is important for patients to have regular defecation habits, reduce mental and physical stress, and improve their diet and lifestyle. ${ }^{15)}$ Laxatives should not be taken for habitual constipation without first trying to improve lifestyle, but should be used only if constipation continues after implementing lifestyle changes. ${ }^{16)}$ Our results showed that those who had consulted a pharmacist were significantly more likely to have bloating, nausea and difficulty in defecating before they took laxatives. This indicated that the patients who consulted a pharmacist took laxatives when their symptoms were worse than those who did not consult. When people select nonprescription medicines, they make their own judgements based on personal values. According to our results (Fig. 4), those values tend to be different in those who consulted a pharmacist before selection. Those who consulted a pharmacist (Group A) were more likely to place importance on preventing interactions, and those who did not (Group B) were more likely to choose a laxative based on manufacturer or brand name, and price. Those who were at risk of unwanted interactions and side effects when they took laxatives were also more likely to consult a pharmacist first. This suggests that they assessed their situation correctly and tried to acquire additional information from the pharmacist before making a decision about whether to take laxatives. Those who consulted a pharmacist were also more likely to make healthy choices in their everyday life. Constipation cannot be managed through medication alone; better short- and long-term outcomes are achieved with lifestyle changes, including proper diet, than with laxatives. ${ }^{17)}$ Those who asked a professional for advice (Group A) were also significantly more likely to be able to identify the side effects of laxatives. It has previously been reported that a lack of knowledge or understanding about medicine does not make people more likely to consult pharmacists. ${ }^{18)}$ Therefore, consultation with a pharmacist, who is specialist for medicine, should be helpful to compensate for a lack of them. Good understanding of medications is necessary to notice side effects and act on them appropriately; we suggest that a prior consultation with a pharmacist is useful in increasing understanding about health and medications.

Appropriate self-medication with nonprescription medicines requires proper information. Although it is best if people seek professional advice about nonprescription medicines every time, it is not always possible. It is therefore important for people to inform themselves about medications. Our results suggest that those who consulted a pharmacist before purchase were also more likely to read a package insert before taking laxatives. This suggests that consultation with the pharmacist was helpful for ensuring proper usage of nonprescription medicines. Those who consulted a pharmacist were also more likely to know how to select laxatives. In contrast, there was no significant difference between the percentages of respondents from both groups who thought that they could select proper laxatives. These results suggest that patients should be able to consult a pharmacist each time they buy laxatives, because these consultations are useful in providing information about how to select laxatives, but insufficient to select them appropriately. Because it is not clear whether pharmacist cannot select appropriate medicine in fact or cannot let patient understand the propriety of their selection for medicine, further study about this will be needed. Anyway, pharmacists also need to improve the quality of consultations for patients to understand how pharmacist selected their medicine and to consent that it has been done appropriately. As described above, lifestyle changes are important in improving constipation and preventing chronic constipation, and laxatives should not be taken until these changes have been made. ${ }^{17)}$ Our results show that those who consulted a pharmacist were more likely to have changed their lifestyle before they took laxatives. This suggests that consultation with the pharmacist may have been helpful in encouraging lifestyle changes, thus preventing chronic constipation.

Our results suggest that it may be important to raise awareness of the benefits of consulting pharmacists. To support this, pharmacists in community pharmacy might undertake a more active role in health promotion campaigns such as drug safety. They could also publicize their role more widely. On the other hand, it is also possible that those who consulted a pharmacist had already made those lifestyle changes before consultation, and were simply more health-conscious. This study could not distinguish between changes made before and after consultation. We still need advanced survey about this.

Our study has several limitations. Since this survey involved monitor-style internet research, one limitation is selection bias because many subjects customarily use the internet for gathering information. In addition, the self-report method is associated with different characteristic measurement errors than other reporting methods like phone surveys. However, bias exists for all questionnaire-bias social research investigations like mail, phone, and interview surveys. ${ }^{19)}$ Therefore, the findings from the present study should be interpreted as the result of monitor-style internet research. Moreover, since this study relied on internet research rather than direct contact with respondents, it is not possible to eliminate the possibility of fraudulent responses like deceit.

\section{CONCLUSION}

We investigated the use of consultations with pharmacists in community pharmacy before selection of nonprescription laxatives in Japan. Our results indicate that it is important that people have the opportunity to consult with pharmacists in community pharmacy, as this will improve awareness of health and appropriate use of medication.

Conflict of Interest The authors declare no conflict of interest.

\section{REFERENCES}

1) Cabinet Office, Government of Japan. "White paper on aging society in 2014.": Retrieved from 〈http://www8.cao.go.jp/kourei/ 
whitepaper/w-2014/zenbun/pdf/1s1s_1.pdf), accessed 5 January, 2016.

2) Prime Minister of Japan and His Cabinet. "Japan Revitalization Strategy—JAPAN is BACK— in 2013.”: 〈http://www.kantei.go.jp/jp/ singi/keizaisaisei/pdf/saikou_jpn.pdf), accessed 5 January, 2016.

3) Ministry of Health. "Labour and Welfare. Investigation of the fixation ratio for the sale system of non-prescription medicines in 2013.": 〈http://www.mhlw.go.jp/bunya/iyakuhin/ippanyou/pdf/ippan-h25.pdf〉, accessed 5 January, 2016.

4) Proprietary Association of Great Britain (PAGB). "A Summary Profile of the OTC Consumer.”: 〈http://www.pagb.co.uk/publications/ pdfs/Summaryprofile.pdf), accessed 28 April, 2016.

5) Wazaify M, Shields E, Hughes CM, McElnay JC. Societal perspectives on over-the-counter (OTC) medicines. Fam. Pract., 22, 170-176 (2005)

6) Izumisawa M, Matubara M, Ikeda S, Sakamaki H, Terasawa T, Yamazaki M. Evaluation on the role of community pharmacist for non-prescription drug consumer satisfaction. Jpn. J. Drug Infom., 4, 27-32 (2002).

7) Sadamoto K, Higashimata Y, Mizoguchi M, Yamanouchi T, Kinoshita N, Saeki T. Expected information of users for the OTC drugs in Japan. Adv. Pharmacoepidemiol. Drug Saf., 1, 1000101 (2012).

8) Sasaki K, Ohbayashi M, Kohyama N, Kobayashi Y, Yamamoto T. Descriptive study on the circumstances concerning confirmation of contraindications and careful administration upon purchasing overthe-counter cold medication and manifestation of after-use urinary disorders. Yakugaku Zasshi, 128, 1301-1309 (2008).

9) Narui K, Ohta J, Yamada Y, Suetsugu D, Watanabe K. Survey of consumer views on non-prescription drugs and self-medication after revised pharmaceutical affairs in 2009. Jpn. J. Drug Inform., 14, 161-169 (2013).
10) Ministry of Health, Labour and Welfare of Japan. "Investigation of the information acquisition for proper usage of non-prescription medicines in 2010.”: 〈http://wwwl.mhlw.go.jp/kinkyu/iyaku_j/ dl/100519j.pdf), accessed 5 January, 2016.

11) Odanaka M, Asahi M, Yokogawa K, Miyamoto K. Investigation of actual use of laxatives and application to the active drug information offer in drug control guidance. Yakugaku Zasshi, 122, 89-95 (2002).

12) Ueki T, Tasaki N, Yoshida T. Relationship among self-evaluated degree of constipation, defecation frequency and use of laxatives. Jpn. J. Pharm. Health Care Sci., 33, 119-124 (2007).

13) Hinkel U, Schuijt C, Erckenbrecht JF. OTC laxative use of sodium picosulfate a results of a pharmacy-based patient survey (cohort study). Int. J. Clin. Pharmacol. Ther., 46, 89-95 (2008).

14) Reisenwitz TH, Wimbish GJ Jr. The purchase decision process and involvement of elderly regarding nonprescription products. Health Mark. Q., 15, 49-68 (1997).

15) Kohashi E. Instruction of life and meal for the constipation patient of women. Medicina, 43, 2035-2037 (2006).

16) Imaizumi Y. Purgatives and laxatives. J. Practic. Pharmacy, 45, 251-255 (1994).

17) Bae SH. Diets for constipation. Pediatr. Gastroenterol. Hepatol. Nutr., 17, 203-208 (2014).

18) Kishimoto K, Fukushima N. Association between understanding proper use of over-the-counter cold medicines and choosing source of information when purchasing these medicines. Jpn. J. Soc. Pharm., 34, 7-19 (2015).

19) Honda N. Quality of web surveys and access panel surveys: issues for utilization of web-based access panel surveys. Jpn. J. Labour Stud., 551, 32-41 (2006). 